\title{
The Associations between Imageability of Positive and Negative Valence Words and Fear Reactivity
}

\author{
Bindiya Lakshmi Raghunath ${ }^{1}$, Claudio Mulatti ${ }^{2}{ }^{\circledR}$, Michelle Jin-Yee Neoh ${ }^{1}\left(D\right.$, Marc H. Bornstein $\left.{ }^{3,4}{ }^{(}\right)$ \\ and Gianluca Esposito 1,2,5,*价 \\ 1 Psychology Program, Nanyang Technological University, Singapore 639818, Singapore; \\ bindiyal001@e.ntu.edu.sg (B.L.R.); michelle008@e.ntu.edu.sg (M.J.-Y.N.) \\ 2 Department of Psychology and Cognitive Science, University of Trento, 38068 Trento, Italy; \\ claudio.mulatti@unitn.it \\ 3 Eunice Kennedy Shriver National Institute of Child Health and Human Development, \\ Bethesda, MD 20892, USA; marc.h.bornstein@gmail.com \\ 4 Institute for Fiscal Studies, London WC1E 7AE, UK \\ 5 Lee Kong Chian School of Medicine, Nanyang Technological University, Singapore 308232, Singapore \\ * Correspondence: gianluca.esposito@ntu.edu.sg or gianluca.esposito@unitn.it
}

Citation: Raghunath, B.L.; Mulatti, C.; Neoh, M.J.-Y.; Bornstein, M.H.; Esposito, G. The Associations between Imageability of Positive and Negative Valence Words and Fear Reactivity. Psychiatry Int. 2021, 2, 32-47. https://doi.org/ 10.3390/psychiatryint2010003

Received: 21 December 2020

Accepted: 5 February 2021

Published: 9 February 2021

Publisher's Note: MDPI stays neutra with regard to jurisdictional claims in published maps and institutional affiliations.

Copyright: (c) 2021 by the authors. Licensee MDPI, Basel, Switzerland. This article is an open access article distributed under the terms and conditions of the Creative Commons Attribution (CC BY) license (https:// creativecommons.org/licenses/by/ $4.0 /)$.

\begin{abstract}
This study investigated the associations of imageability with fear reactivity. Imageability ratings of four word classes: positive and negative (i) emotional and (ii) propriosensitive, neutral and negative (iii) theoretical and (iv) neutral concrete filler, and fear reactivity scores-degree of fearfulness towards different situations (Total Fear (TF) score) and total number of extreme fears and phobias (Extreme Fear (EF) score), were obtained from 171 participants. Correlations between imageability, TF and EF scores were tested to analyze how word categories and their valence were associated with fear reactivity. Imageability ratings were submitted to recursive partitioning. Participants with high TF and EF scores had higher imageability for negative emotional and negative theoretical words. The correlations between imageability of negative emotional words and negative theoretical words for EF score were significant. Males showed stronger correlations for imageability of negative emotional words for EF and TF scores. High imageability for positive emotional words was associated with lower fear reactivity in females. These findings were discussed with regard to negative attentional bias theory of anxiety, influence on emotional systems, and gender-specific coping styles. This study provides insight into cognitive functions involved in mental imagery, semantic competence for mental imagery in relation to fear reactivity, and a potential psycholinguistic instrument assessing fear reactivity.
\end{abstract}

Keywords: mental imagery; fear reactivity; emotion recognition; emotion regulation; propriosensitive

\section{Introduction}

The imageability scale is a classical psycholinguistic measure for assessing the wordspecific semantic feature that refers to the degree to which words evoke mental images [1,2]. Mental images are perceptual representations that codify past sensory experience. Mental imagery allows for conscious recall of past experiences and reenacts them as visual scenes, sounds, smells, as well as sensations of any kind in the absence of the stimuli [3]. As Paivio stated, "imagery can and does occur as an associative reaction to words, and [... ] it plays a part in our memory for (and comprehension of) language" [4].

Different words can have stronger or weaker links to sensory experience, and thus differ in imageability ratings on a seven-point Likert scale. Words that rely heavily on external sensory information (i.e., concrete words) and internal sensory information (i.e., words describing proprioceptive, interoceptive and emotional states) have high imageability as they are more directly connected to sensory and perceptual experience (external or internal) [5]. These words arouse mental images easily relative to words that rely more 
heavily on linguistic information, such as, abstract or theoretical words that have low imageability due to difficulty in picturing the words [6-13]. High-imageability words are processed and understood more easily than low-imageability items $[1,4,14,15]$.

Individual differences in imageability exist such that different people exhibit more ease or difficulty in recalling sensory experience (for a review of some classical studies on this topic see, e.g., $[3,16,17])$. This variance may be due to their specific cognitive style determined by, or at least correlated with factors related to, personality (see [18,19]). Sensory processing sensitivity is one such personality trait that involves being more or less responsive, reactive or receptive, to some environmental stimuli (for a review see e.g., $[20,21])$ : people with high sensory processing sensitivity process information more strongly and deeply, and perceptively retain this information more easily than others with low sensory processing sensitivity. Thus, they have a higher imagery capacity for particular word items compared to people with low sensory processing sensitivity.

\subsection{Association of Imageability with Fear Reactivity}

In this study, we assessed how imageability is associated with individuals' fear reactivity. Fear is a response to stimuli that are perceived as threat. It may manifest as overt behavioral responses, physiological, and cognitive responses [22,23]. Overt behavioral responses of fear include actions employed to avoid or reduce contact with feared object. Physiologically, fear reactions include heart palpitations and sweating. Finally, examples of cognitive fear responses constitute feelings of distress or anxiety of feared objects [23]. In the present study, the Fear Survey Schedule-II (FSS; [24]) was used to measure fearfulness and number of extreme fears in individuals. FSS-II was developed to measure phobic behavior or extreme fear, and generalized anxiety in experimental studies [24].

There is scarce literature on imageability and fear reactivity or anxiety. Ferguson et al. [25] showed individuals with high scores on health anxiety on Whitely Index - a questionnaire designed to assess hypochondriasis-reported higher imageability and higher negative emotional valence for health-related words [25].

It should be noted here that anxiety is positively correlated to high sensory processing sensitivity (see, e.g., [21,26-30], which may explain Ferguson et al.'s [25] finding that people with high health anxiety have higher imageability scores specifically for health related words [25]. Thus, it is possible that the imageability scores of different word categories might serve as an index for measuring anxiety levels, especially, fear reactivity (fearfulness towards situations and total number of extreme fears).

\subsection{Current Study}

Specifically, we investigated whether imageability ratings of different word categories were associated with an individual's fearfulness towards different situations (fear tendency) and their total number of extreme fears, phobias. Individuals' scores for fearfulness towards situations and total number of extreme fears fall on a continuum. A correlation between imageability and fearfulness will provide support for a new psycholinguistic instrument assessing fear reactivity as well as important cues regarding cognitive mechanisms and semantic competence of persons with more or less marked fear reactivity. Specifically, this study might allow us to understand how perceptual representations of different word categories would associate with varying degrees of fear reactivity.

This study also considered gender differences in the association of imageability of different word categories and fear reactivity. Abundant literature suggests females were more likely to report higher fear ratings, were more vulnerable to various anxiety disorders, and use different coping strategies compared to their male counterparts (for e.g., see [31-36]). Although extant literature suggest that females construct more coherent and vivid mental simulations (for e.g., see [37], no gender differences in mental imagery or imageability was previously reported $[38,39]$. Thus, investigating any gender differences would provide an opportunity to understand its influences on the association of imageability of different word categories with fear reactivity. 
Four word categories, adapted from [40], were used in this study: two classes represented internal states, (i) emotional words and (ii) propriosensitive words; the third category consisted of (iii) theoretical words; and the last category consisted of (iv) concrete filler words. Concrete filler words were matched for frequency and letter length with the rest of the other words to serve as fillers. (i) Emotional words constituted of words that denote emotional states including primary and secondary emotions, as well as moods. (ii) Propriosensitive words described internal sensations of proprioceptive and interoceptive kind, i.e., related to body positions and muscle tension (e.g., 'spasm', 'convulsion' or 'relaxation', 'balance') and to the viscera, internal organs, temperature, pain, vasomotor activity, etc. (e.g., 'cold', 'pain', 'pale', 'nausea'). (iii) Theoretical words in this study were identified to indicate a particular sub-class of abstract words. Typically, words are qualified as abstract when their meaning is not determined on the basis of sensory information but is fixed definitionally by inferential relationships with other words. However, abstract words are not a homogeneous class. A number of them include emotional information (see, e.g., [41]). To avoid this bias, we built a class of theoretical words which only include words that rely substantially on linguistic information, i.e., on definitions. This was composed based on technical terms driven by various scientific disciplines (e.g., 'function', 'fallacy', method', etc., see [5]). (iv) The fourth category comprised of concrete fillers. The concrete words rely on external sensory information, such as, 'armchair', 'car', 'dictionary', etc. Emotional, propriosensitive, and theoretical words had two subgroups of words based on their valence. Emotional and propriosensitive word categories had words with positive and negative valence. Theoretical word category had words with neutral and negative valence. Concrete fillers only consisted of neutral words.

To understand the association of imageability and fear reactivity, we first tested for correlations between the imageability ratings of the four word categories and their valence with fear reactivity scores: the degree of fearfulness towards different situations (fear tendency/Total Fear score; TF) and total number of extreme fears or phobias (Extreme Fear; EF), Extending from the finding that showed people with high anxiety have high sensory processing sensitivity, we expect that people with high fear reactivity scores will have higher imageability ratings on all word categories denoting emotions, sensations or even situations and conditions characterized by a negative hedonic tone (i.e., that feels unpleasant to whom experiences them). These correlations were conducted for the whole-group and separately for males and females to assess any gender differences.

To further explore how imageability scores of different word categories and their valence predict fear reactivity scores, we employed a novel analytic path, exploratory recursive partitioning (regression tree-based models). Specifically, we investigated (1) how imageability of different word categories and their valences predicted (i) TF in males, (ii) TF in females, (iii) EF in males, and (iv) EF in females. Additionally we also investigated (2) how imageability of individual words (regardless of their category) predicted (i) TF in males, (ii) TF in females, (iii) EF in males, and (iv) EF in females. Owing to the existing literature on gender differences we expected different hierarchical patterns of word categories and individual words in predicting fear reactivity for males and females.

\section{Material and Methods}

\subsection{Sample}

Data from 171 participants were collected through Google Form Online Survey and were analysed for this study. The sample consisted of 103 female (Mean Age $=30.72$ years, $S D=11.00$ ) and 68 male (Mean Age $=33.12$ years, $S D=10.86$ ) participants The age of the same ranged from 18 years to 58 years. Their educational level was medium-high (with all the participants having at least completed high school). All participants were Italian, hence all the questionnaires were administered in Italian. Participants were provided with information on the basic background of the study and the procedure before giving their consent. Participants did not receive any monetary incentive to participate in the study. The study was approved by the Internal Ethical Committee of the University of Padova, 
and was conducted according to the principles expressed in the Declaration of Helsinki. Informed consent was obtained from the participants.

\subsection{Words}

A total of 180 Italian words were selected as experimental stimuli. These words belonged to one of the three categories: emotional words $(N=60)$, propriosensitive words $(N=60)$, and theoretical words $(N=60)$. Each word category consisted of negative, and positive, or neutral valence words. An additional 180 words were selected as concrete fillers. Experimental words and concrete fillers were balanced based on written frequency $(67.2 \mathrm{vs}$. 67.3 , respectively; $t<|1|)$ and letter length $(8.5$ vs. $8.5 ; t<|1|)$ of words. (i) Emotional words consisted of both transient emotions and moods. The list was composed based on examples from previous studies using emotional words. We included only emotional words that are considered to be stable across different cultures [42-49]. As for moods, the list was composed based on the cases discussed in $[11,45,50]$. Examples of words included 'anguish', 'amazement', 'joy', 'happiness', 'sadness', 'unhappiness', 'disappointment', 'love', 'fear', 'anger', 'depression', 'boredom', 'distress', 'indolence', 'relief'. (ii) Words denoting propriosensitive states were selected based on the words used in studies by [51-54]. Some examples are 'agitation', 'balance', 'blush', 'cramp', 'spasm', 'pain', 'hunger', 'cold', 'fullness', 'fever', 'malaise', 'relaxation', 'wheeze'. (iii) The list of theoretical words was not derived from previous databases of abstract words. This is because many words that are considered abstract may be linked to internal-emotional or propriosensitive-sensory information (e.g., 'friendship', 'love', 'freedom', or 'wellness', etc.; on this see also [12,13]). Therefore, the class of theoretical words was selected with the focus on obtaining a set of terms which rely mainly on verbal definitions like 'axiom', 'fallacy', hypothesis', 'fraud', 'democracy', 'definition', 'exception', 'unanimity' 'protocol', etc. (iv) The concrete fillers (matched for length and frequency with the other three groups) were composed uniquely of concrete words, i.e., words which strongly rely on external sensory information. Words such as 'table', 'fennel', 'candle', 'juice', 'cork', 'pillow', 'melon', 'book', 'stem glass', and 'airplane' were included in this class (see [40]).

For (i) and (ii), positive and negative words were distinguished based on their valence. Emotional words such as 'happiness', 'satisfaction', 'thrill', 'pleasure', 'relief', etc. and to propriosensitive words such as 'relaxation', 'balance', 'sit', 'excitement', 'sleep', 'jump', etc., which have a positive valence comprised of the positive words. They were contrasted to emotional words such as 'apathy', 'remorse', 'anxiety', 'anguish', 'stress', etc. and propriosensitive words such as 'spasm', 'convulsion', 'pale', 'burn', 'unease', 'nausea', etc., which were considered negative as they have a negative hedonic tone. (iii) As for theoretical words, most of them have a neutral valence (e.g., 'hypothesis', 'property', 'parallelism', 'chronology', 'idiom', etc.) since they denote something that does not affect us in any way. However, some refer to situations or conditions that are problematic or give rise to negative consequences or feelings (e.g., 'abstruse', 'fallacy', 'incongruence', 'fraud', 'contamination', etc.). On this basis, theoretical words were divided into neutral and negative words based on valence. The list consisted of words such as 'hypothesis', 'property', 'parallelism', 'chronology', 'idiom', etc. These words do not have a specific affect. Negative theoretical words refer to situations or conditions that are problematic or give rise to negative consequences or feelings (e.g., 'abstruse', 'fallacy', 'incongruence', 'fraud', 'contamination', etc.). (iv) Concrete fillers were not distinguished as positive and negative, but were considered as a uniformly neutral class. Two research assistants judged the valence of the words independently and their agreement was calculated using Cohen Kappa ( $>0.98)$. As standard procedure in the field, valence scores of these words were also checked with the scores from [55] Warriner et al.'s (2013) database. Positive emotional words ( $M$ valence $=7.15, S D=0.584$ ) had significantly higher valence than negative emotional words $(M$ valence $=3.03, S D=0.703) ; t(50,2$-tailed $)=21.87$, $p<0.01$. Positive propriosensitive words $(M$ valence $=6.13, S D=1.45)$ had significantly higher valence than negative propriosensitive words $(M$ valence $=3.80, S D=1.26) ; t(54,2$-tailed $)=$ $6.30, p<0.00$. Negative theoretical words ( $M$ valence $=3.47, S D=0.80$ ) had a significantly 
lower valence than neutral theoretical words $(M$ valence $=5.32, S D=0.71) ; t(46,2$-tailed $)=$ $-7.56, p<0.00$. Valence of all negative words ( $M$ valence $=3.44, S D=1.10$ ) was significantly lower than all positive words ( $M$ valence $=6.64, S D=1.21$ ). Positive and negative words were significantly different from neutral words $(M$ valence $=5.73, S D=0.92)$. There was no significant difference in arousal scores of positive ( $M$ arousal $=5.04, S D=1.37)$ and negative emotional $(M$ arousal $=4.99, S D=1.16)$ words, for positive $(M$ arousal $=4.08, S D=1.23)$ and negative propriosensitive $(M$ arousal $=4.63, S D=0.68)$ words, and for neutral $(M$ arousal $=$ $4.07, S D=0.66)$ and negative theoretical $(M$ arousal $=4.67, S D=1.16)$ words; $p<0.01$. Arousal scores for neutral words ( $M$ arousal $=3.91, S D=0.81$ ) were significantly different from arousal scores for positive ( $M$ arousal $=4.56, S D=1.38$ ) and negative words (mean arousal $=4.78$, $S D=0.98) ; p<0.01$. As a part of our analyses, all negative words and all positive words were combined respectively from all words categories. Neutral theoretical words were included in the positive words category as they were significantly more positive ( $M$ valence $=$ $5.32, S D=0.71)$ than negative words $(M$ valence $=3.44, S D=1.05) ; t(114,2$-tailed $)=-9.72$, $p<0.01)$.

\subsection{Fear Survey Schedule—Revised (FFS-R). Cognitive Behaviour Assessment (CBA) Schedule 7}

Participants were administered the full 58-item FSS-R [24] from the Cognitive Behaviour Assessment Battery (CBA)—Schedule 7 [56]. Answering each question on the survey was mandatory, so there were no missing data for any participants who completed it. FSS-R results were scored according to Sanavio et al. 1997's scoring manual for CBA Schedules [57]. The two main scores of interest are TF and EF scores. The TF scores represent individual's tendency to react with fear to different situations. The EF score indicates the exact number of situations that induce a fear or phobic response (total number of situations for which an individual lies in the far high end of the fear Likert scale).

\subsection{Procedure}

The survey was conducted between July 2014 to November 2014 using Google Forms Online Surveys. Participants were asked to complete the FSS-R section of the CBA questionnaire and to rate the imageability of a set of 360 words. Imageability was rated for each word on a 7-point Likert scale ( $1=$ low imageability to $7=$ high imageability). Instructions to participants were in Italian which were based on a translation of Paivio and colleagues' original instructions [58]: "Nouns differ in their capacity to arouse mental images of things or events. Some words arouse a sensory experience, such as a mental picture or sound, very quickly and easily, whereas others may do so only with difficulty (i.e., after a long delay) or not at all. The purpose of this experiment is to rate a list of words as to the ease or difficulty with which they arouse mental images. Any word which, in your estimation, arouses a mental image (i.e., a mental picture, or sound, or other sensory experience) very quickly and easily should be given a high imagery rating: any word that arouses a mental image with difficulty or not at all should be given a low imagery rating. Think of the words 'apple' or 'fact'. Apple would probably arouse an image relatively easily and would be rated as high imagery; fact would probably do so with difficulty and would be rated as low imagery." (see [58], p. 4; for an analysis of these instructions and how people might plausibly interpret them to assign ratings, see [59].

The experiment consisted of two parts. In the first part, participants were asked to complete the CBA questionnaire and ratings of a random selection of 180 words; in the second part, they were asked to rate the remaining 180 words. Only data from participants ( $95 \%$ of the total sample) who completed the questionnaire and ratings of both word blocks were used for the analyses.

\subsection{Analytic Plan}

In the preliminary analyses, $\mathrm{TF}, \mathrm{EF}$, and imageability scores were checked for normalcy, homogeneity of variance, outliers, and influential cases [60]. Correlations among TF and 
EF scores were assessed. Gender differences for TF, EF, and imageability scores were also assessed.

To understand the relationship between fear reactivity scores and imageability, correlations between TF and imageability, and EF and imageability scores were conducted for each word category (positive and negative emotional words, positive and negative propriosensitive words, neutral and negative theoretical words, and neutral concrete words). These correlations were conducted for the whole group and separately for males and females to determine any gender differences.

To further explore how imageability scores of different word categories and their valence predict fear reactivity scores, we employed a novel analytic path, exploratory recursive partitioning (regression tree-based models). Specifically, we investigated (1) how imageability of different word categories and their valences predicted (i) TF in males, (ii) TF in females, (iii) EF in males, and (iv) EF in females. Additionally we also investigated (2) how imageability of individual words (regardless of their category) predicted (i) TF in males, (ii) TF in females, (iii) EF in males, and (iv) EF in females.

Recursive partitioning explores data relations with easy-to-visualize decision rules to predict a continuous outcome [61,62]. These tree-based models uncover the hierarchical importance of independent variables that explain variance in the dependent variable along with the value of the independent variable that significantly stratifies the dependent variable into two parts. This is achieved in two steps: first, the growing step in which the model explores all possible relations among variables, and second, the pruning step during which the independent variable (i.e., imageability scores) stratify the dependant variable (i.e., fear reactivity scores) into two subsamples that differ significantly. Recursive partitioning was calculated using the rpart package of statistical software R (ver. 4.1-13) with the minimum number of observations in any terminal node set to 15 . The tree allowed for maximum growth by setting the complexity parameter close to zero at 0.0002 and was subsequently pruned to obtain an optimal tree by removing branches containing nodes with $\mathrm{t}$-values greater than $1.64(\alpha=0.05)$.

\section{Results}

\subsection{Preliminary Analysis}

The data were explored and analysed using R-project (ver. 4.1-13). Prior to data analysis, univariate and multivariate distributions of TF, EF, and imageability judgment scores were examined for normalcy, homogeneity of variance, outliers, and influential cases [63]. All these variables, except EF scores, were normally distributed. EF scores were distributed as a non-continuous variable and showed a significantly skewed distribution $(p<0.01)$. This variable did not satisfy the normalcy assumptions and was therefore treated as an ordinal (rather than interval) variable. The distance of each case to the centre was evaluated to screen for multidimensional outliers [63]. TF scores and EF scores were positively correlated to each other, $\mathrm{r}=0.69, p<0.001$, sharing however, only $47.3 \%$ of their common variance. There were no significant gender difference for TF (male $M=67.1 ; S D=30.8$; female $M=68.7 ; S D=27.0 ; F(1,169)=0.13 ; n s)$ and EF scores (male $M=3.67 ; S D=4.71$; female $M=4.29 ; S D=4.59 ; F(1,169)=0.75 ; n s$ ). Similarly, no significant differences emerged between males and females for the imageability scores ( $F$ range $=0.16-1.6 ; n s)$.

\subsection{Correlational Analysis}

For the whole group, there was a significant positive correlation of TF score and imageability ratings of negative emotional words $(r=0.18, p=0.02)$, and negative theoretical words $(r=0.18, p=0.02)$. Only males (not females) showed a significant positive correlation of TF scores with imageability ratings of negative emotional words $(r=0.27, p=0.02)$. For EF scores of the whole group, there was a significant positive correlation with imageability of negative emotional words (Kendal's Tau $=0.14, p=0.01$ ), and negative theoretical words (Kendal's Tau $=0.13, p=0.02$ ). There was also a significant positive correlation of EF 
score and imageability of negative words from all experimental word clusters combined (emotional, propriosensitive, theoretical; Kendal's Tau $=0.11, p=0.03$ ), and for negative theoretical words (Kendal's Tau $=0.13, p=0.02$ ). Only males (but not females) showed a significant positive correlation of EF scores with imageability of negative emotional words (Kendal's Tau $=0.24, p<0.01$ ), positive theoretical words (Kendal's Tau $=0.20, p=0.02$ ), and negative theoretical words (Kendal's Tau $=0.21, p=0.02$ ). There was a significant positive correlation of EF scores of males with negative words from all experimental word clusters (Kendal's Tau $=0.20, p=0.02$ ), positive theoretical words (Kendal's Tau $=0.20$, $p=0.02$ ), and negative theoretical words (Kendal's Tau $=0.21, p=0.02$ ). Figure 1 shows the linear correlations of TF scores (Figure 1A) and EF scores (Figure 1B) with imageability ratings for word clusters that were significant in either male or female participants.
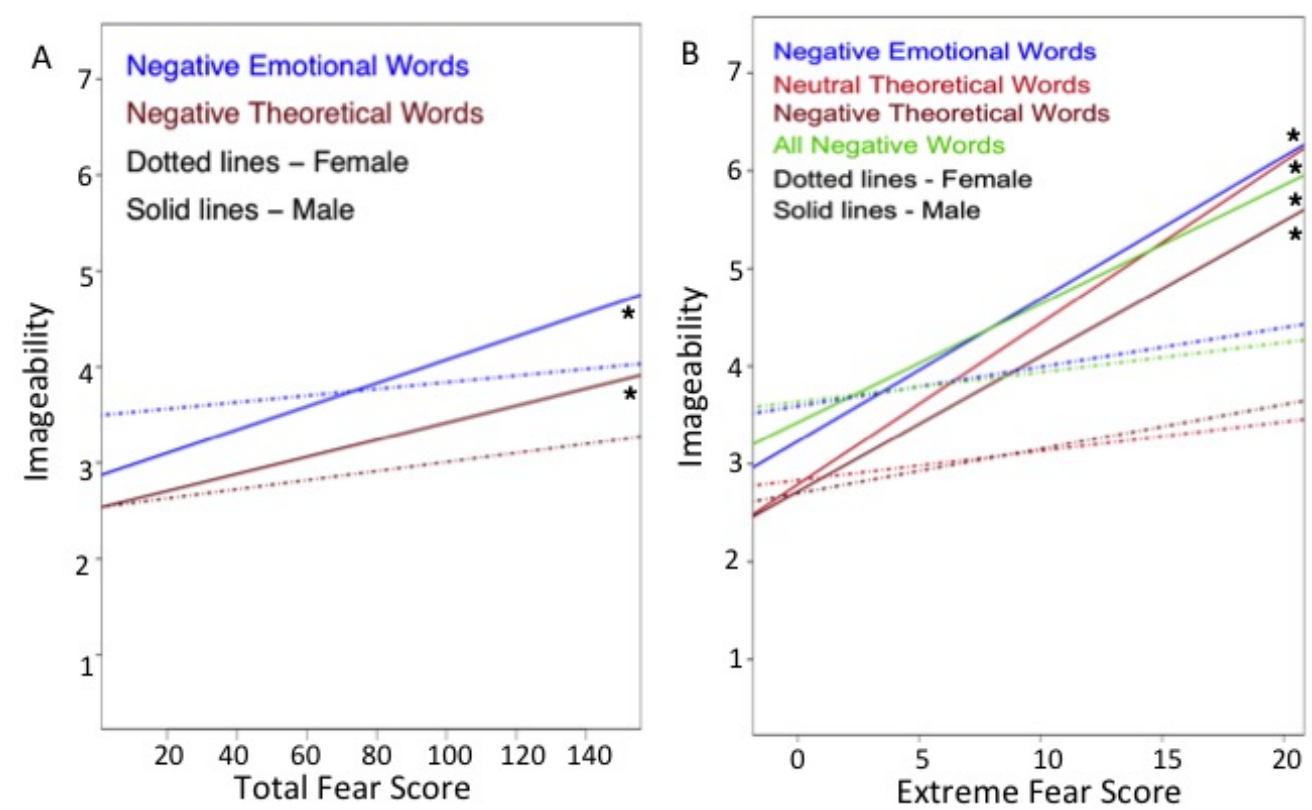

Figure 1. (A) shows the correlations between imageability of (i) negative emotional and (ii) negative theoretical words with Total Fear Scores (TF) scores. Only the correlation between the imageability of negative emotional words and TF scores was significant. (B) shows the correlations between imageability of (i) negative emotional, (ii) neutral theoretical, (iii) negative theoretical and (iv) all negative words with Extreme Fear (EF) scores. Only males (but not females) showed a significant positive correlation of EF scores with imageability of negative emotional words, positive theoretical words, and negative theoretical words. ${ }^{*} p<0.05$.

\subsection{Tree-Base Model Analysis \\ 3.3.1. Category-Words Level}

Figure 2 shows the optimal tree that describes how imageability ratings of the word categories and their valence predicted the TF subscale of CBA scores for males and females. For males, only imageability of negative emotional words partitioned TF score: males that reported greater imageability for negative emotional words ( $>3.67$, i.e., the 'no' branch of the Negative Emotional <3.67 leaf) had higher TF scores (77.4). For females, the path to higher TF score led from imageability of all negative words to imageability of only negative emotional words. In contrast, the path to lower TF score led from imageability of all negative words to imageability of only positive emotional words. Thus, imageability of all negative words combine from all experimental word categories first partitioned TF score. Amongst females who reported greater imageability for all negative words combine $(>2.69$, i.e., the 'no' branch of the All Negative < 2.69 leaf), imageability of negative emotional words further partitioned TF score: females who reported greater imageability of negative emotional words ( $>4.70$, i.e., the 'no' branch of the Negative Emotional $<4.70$ leaf) had higher TF scores (80.6). Amongst females who reported lower imageability of all negative words combine 
( $<2.69$, i.e., the 'yes' branch of the All Negative $<2.69$ leaf), imageability of positive emotional words further partitioned TF score: females who reported greater imageability of positive emotional words ( $\geq 3.68$, i.e., the 'yes' branch of the Positive Emotional $\geq 3.68$ leaf) had lower TF scores (45.5). Figure 2B shows the optimal tree that described how imageability rating of the word categories and their valence predicted the EF subscale of CBA scores for males and females. For males, imageability of all negative words combine and imageability of negative propriosensitive words predicted EF scores. Thus, imageability of all negative words combine first partitioned EF score: males who reported greater imageability of all negative words ( $>4.78$, i.e., the 'no' branch of the All Negative $<4.78$ leaf), had higher EF scores (7.31). Amongst males who reported lower imageability of all negative words $(<4.78$, i.e., the 'yes' branch of the All Negative < 4.78 leaf), imageability of negative propriosensitive words further partitioned EF score: males who reported greater imageability of negative propriosensitive words ( $\geq 3.82$, i.e., the 'yes' branch of the Negative Propriosensitive $\geq 3.82$ leaf), had the lowest EF scores (1.37) as opposed to men who reported lower imageability of negative propriosensitive words $\left(\leq 3.82\right.$, i.e., the ' $n o^{\prime}$ branch of the Negative Propriosensitive $\geq 3.82$ leaf), who had higher EF scores (4.14). For females, imageability of all negative words combine, all positive words combine, neutral theoretical words, and negative emotional words predicted EF score. Of interest, imageability of all negative words combine first partitioned EF scores: females who reported greater imageability of all negative words ( $>4.78$, i.e., the ' $n o$ ' branch of the All Negative < 4.78 leaf) had higher EF scores (6.96). Females who reported lower imageability of all negative words $(<4.78$, i.e., the 'yes' branch of the All Negative $<4.78$ leaf $)$ and greater imageability of all positive words $(\geq 4.48$, i.e., the 'yes' branch of the All Positive $\geq 4.48$ leaf), had the lowest EF scores (1.07).

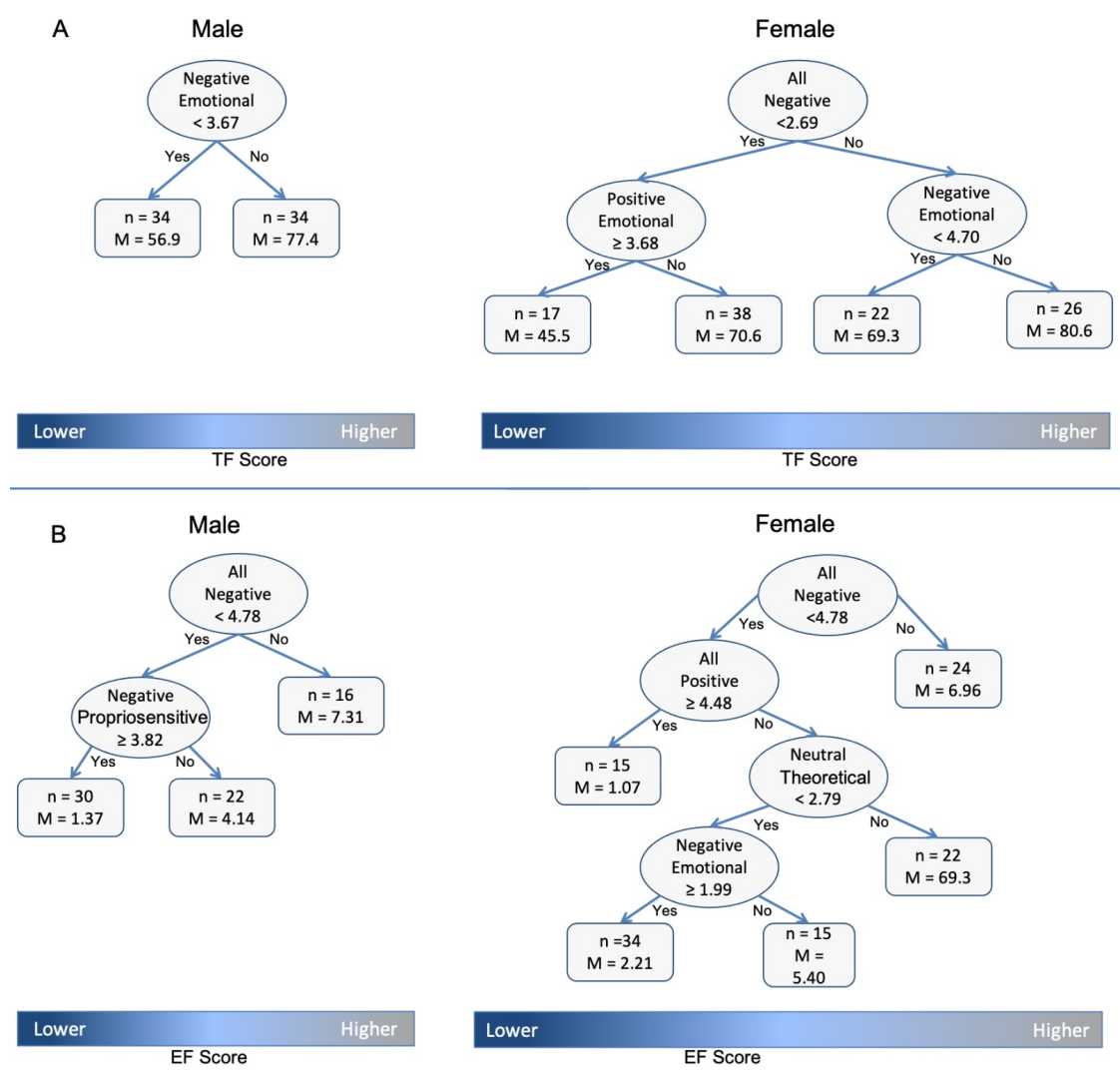

Figure 2. The optimal tree that describes how imageability ratings of word categories predict the TF (A) and EF (B) scores for males and females. The bottom rectangle shows the distribution of the TF (A) or EF (B) scores from lower (left) to higher (right) scores. The values in oval leaves of the tree refer to the condition of the independent variables (positive and negative emotional words, positive and negative propriosensitive words, neutral and negative theoretical words, and neutral concrete filler words) that statistically divides the distribution of the dependent variable (fear reactivity score-either 
TF or EF). Below each oval leaf, the indications "yes" or "no" refer to whether or not the condition is met. Each leaf is divided in two sub-leaves. The terminal leaves (quadrangles) represent subgroups that cannot be further subdivided. The $\mathrm{N}$ value in the terminal leaves represents the size of the group, and $\mathrm{M}$ is the mean value of the group for the dependent variable.

\subsubsection{Single-Word Level}

Figure 3A shows the optimal tree that describes how imageability ratings at the single word level (all 360 words were considered independently) predict the TF subscales of the CBA for males and females. For males, imageability of the word 'anxiety', belonging to the negative emotional word category, first partitioned TF scores: Males who reported higher imageability for the word 'anxiety' ( $>4.75$, i.e., the 'no' branch of the Anxiety $<4.75$ leaf) had higher TF scores (82.3). Amongst males who reported lower imageability for the word 'anxiety' $(<4.75$, i.e., the 'yes' branch of the Anxiety $<4.75$ leaf $)$, the word 'puzzle', belonging to the neutral theoretical word category, further partitioned the TF score: males who reported greater imageability of the word 'puzzle' ( $\geq 5.50$, i.e., the 'yes' branch of the Puzzle $\geq 5.50$ leaf) had lower TF scores (38.1). For females, a neutral theoretical word 'convocation' best stratified the TF scores. Females who reported low imageability for the word 'convocation' ( $<2.69$, i.e., the 'yes' branch of the Convocation $<2.69$ leaf ) had lower TF scores (59.6). Amongst females who reported greater imageability of the word 'convocation', the imageability of the positive propriosensitive word 'salivation' further partitioned TF score: females who reported lower imageability for the word 'salivation' ( $\leq 5.50$, i.e., the 'no' branch of the Salivation $\geq 5.50$ leaf) had higher TF scores (87.1).

Figure $3 \mathrm{~B}$ shows the optimal tree that describes how imageability ratings at the single word level (all 360 words were considered independently) predict the EF subscales of the CBA for males and females. For males, imageability ratings of the word 'frustration', a negative emotional word, first partitioned $\mathrm{EF}$ score: males with greater imageability for the word 'frustration' ( $>4.50$, i.e., the 'no' branch of the Frustration $<4.50$ leaf) had higher EF scores. Amongst males who reported lower imageability of the word 'frustration' $(<4.50$, i.e., the 'yes' branch of the Frustration < 4.50 leaf), imageability of the words 'traffic' (negative theoretical word), and 'pinch' (negative propriosensitive word) further partitioned the EF score: males who reported higher imageability for 'traffic' $(\geq 4.50$, i.e., the 'yes' branch of the Traffic $\geq 4.50$ leaf), and 'pinch' ( $\geq 5.50$, i.e., the 'yes' branch of the Pinch $\geq 5.50$ leaf) had the lowest EF scores (0.30). For females, imageability of the word 'contamination' (negative theoretical word) first partitioned the EF score: females who reported greater imageability of the word 'contamination' ( $>6.50$, i.e., the 'no' branch of the Contamination $<6.50$ leaf) had higher EF scores (10.8). Amongst females who reported lower imageability of the word 'contamination' ( $<6.50$, i.e., the 'yes' branch of the Contamination < 6.50 leaf), imageability of the words 'orientatation' (neutral theoretical word) and 'trailer' neutral theoretical word), followed by 'joy' (positive emotional word) and 'definition' (<3.5) further stratified the EF score: females who reported greater imageability for the word 'orientation' $(\geq 1.50$, i.e., the 'yes' branch of the Orientation $\geq 1.50$ leaf $)$, lower imageability for the word 'trailer' $(<6.50$, i.e., the 'yes' branch of the Trailer $<6.50$ leaf), and greater imageability for the word 'joy' ( $\geq 4.58$, i.e., the 'yes' branch of the Orientation $\geq 4.58$ leaf) had the lowest EF scores (0.57). 


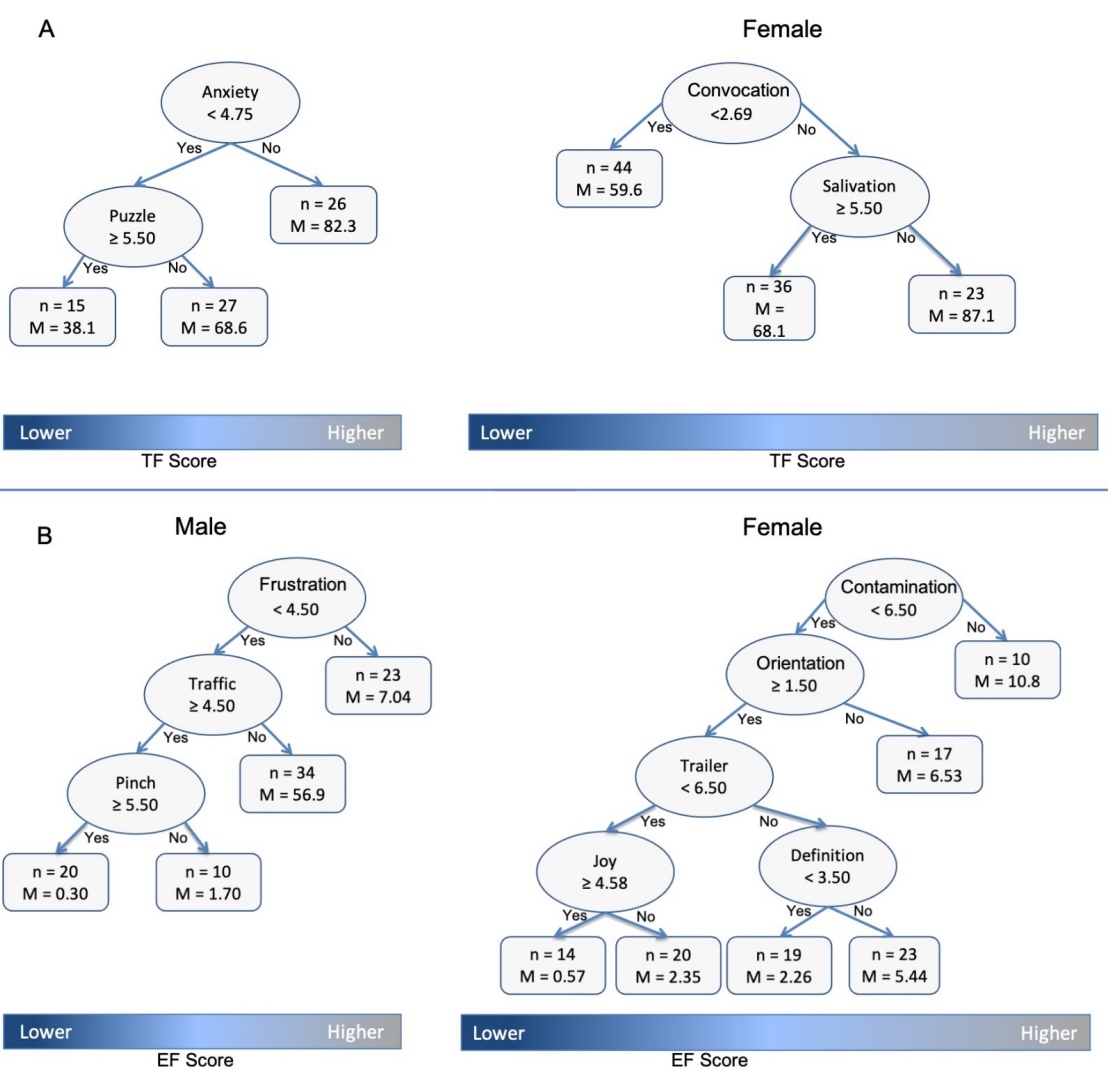

Figure 3. The optimal tree that describes how imageability ratings of single words predict the TF (A) and EF (B) scores for males and females. The bottom rectangle shows the distribution of the TF (A) or EF (B) scores from lower (left) to higher (right) scores. The values in oval leaves of the tree refer to the condition of the independent variables (positive and negative emotional words, positive and negative propriosensitive words, neutral and negative theoretical words, and neutral concrete filler words) that statistically divides the distribution of the dependent variable (fear reactivity score-either TF or EF). Below each oval leaf, the indications "yes" or "no" refer to whether or not the condition is met. Each leaf is divided in two sub-leaves. The terminal leaves (quadrangles) represent subgroups that cannot be further subdivided. The $\mathrm{N}$ value in the terminal leaves represents the size of the group, and $\mathrm{M}$ is the mean value of the group for the dependent variable.

\section{Discussion}

This study addressed how imageability of specific word categories and their valence (positive and negative emotional words, positive and negative propriosensitive words, neutral and negative theoretical words, and neutral concrete filler words) is associated with the varying degree of fear reactivity (the degree of fearfulness towards different situations/fear tendency-TF, and total number of extreme fears or phobias-EF) in males and females. This study sought to provide insights into underlying cognitive functions involved with representations and semantic competence of mental imagery in individuals with high or low fear reactivity. This otherwise would have been difficult to conceptualize owing to the subjective and private nature of this phenomenon.

In accordance to our expectations that imageability of negative words would be associated with fear reactivity, results showed that imageability of negative emotional words and negative theoretical words were positively correlated with greater fear towards different situations and higher number of extreme fears or phobias. Additionally imageability of all negative words combine from all word categories (emotional, propriosensitive, and theoretical) was positively correlated to higher number of extreme fears or phobias. These correlations were only evident in males upon examining separately for males and females, except the positive correlation between imageability of negative theoretical words and 
greater fear tendency, which only existed in the whole-group analysis. Although there was no difference in fear reactivity and imageability scores across males and females in our sample, there was differing associations of imageability of specific word categories with fear reactivity amongst males and females.

The tree-based models provided further details regarding the hierarchical relationship of the imageability of specific word categories and individual words with fear reactivity in males and females. As expected this hierarchical relationship varied across males and females. In males, greater imageability of negative emotional words best predicted fear tendency, whereas in females, greater imageability of all negative words combine from all categories best predicted fear tendency, followed by imageability of negative emotional words (Figure 2A). Additionally in females, greater imageability of all positive words predicted low fear tendency.

Only the first partitioning of the tree models predicting extreme fears and phobias were similar in males and females. Greater imageability of all negative words best predicted high number of extreme fears and phobias in both males and females. In males, imageability of negative propriosensitive words further predicted extreme fears and phobias; lower imageability of negative propriosensitive words predicted higher number of extreme fears and phobias whereas high imageability of negative propriosensitive words predicted lower number of extreme fears and phobias. In females, imageability of all positive words further predicted extreme fears and phobias; greater imageability of all positive words predicted lower number of extreme fears and phobias (Figure 2B).

The hierarchical relationship of the imageability of individual words with fear reactivity also differed amongst males and females. In males, 'anxiety', belonging to the negative emotional category, best predicted fear tendency, followed by 'puzzle', belonging to the neutral theoretical category. High imageability of the word 'anxiety' predicted high fear tendency, whereas low imageability of the word 'anxiety' and high imageability of the word 'puzzle' predicted low fear tendency. On the other hand, imageability of the word 'convocation', belonging to the neutral theoretical category, best predicted fear tendency amongst females, followed by the word 'salivation', belonging to the positive propriosensitive category. Lower imageability of the word 'convocation' predicted low fear tendency, whereas high imageability of the word 'convocation' and low imageability of the word 'salivation' predicted high fear tendency. In males, imageability of the word 'frustration', belonging to the negative emotional word category best predicted extreme fears and phobias, followed by the words 'traffic', belonging to the negative theoretical word category, and 'pinch', belonging to the negative propriosensitive category. Greater imageability of the word 'frustration' predicted higher number of extreme fears and phobias, whereas, lower imageability of the word 'frustration' and higher imageability of the words 'traffic' and 'pinch' predicted lower number of extreme fears and phobias. On the other hand, amongst females, imageability of the word 'contamination', belonging to the negative theoretical category, best predicted extreme fears and phobias, followed by the words 'orientation' and 'trailer', both belonging to neutral theoretical category, and 'joy', belonging to the positive emotional category. Higher imageability of the word 'contamination' predicted higher number of extreme fears and phobias, whereas, lower imageability of the word 'contamination', higher imageability of the word 'orientation', followed by lower imageability of the word 'trailer' and higher imageability of the word 'joy' predicted lower number of extreme fears and phobias.

Overall, these results are consistent with the negative attentional bias theory of anxiety (for review see [64]). Literature has shown that anxious individuals selectively attend to the threat-related information in the environment [64-67]. Thus, participants with high fear reactivity may have enhanced mental imagery for negative words. This could be seen in males and females where higher imageability of either negative emotional words (in males) or all negative words (in females) were the best predictors of fear tendency and extreme fears and phobias (except in the tree model predicting fear reactivity using single words in females). Additionally, increased imageability for emotional words with negative valence 
corroborates the notion that mental imagery has a powerful impact on emotion in at least three ways: a direct influence on emotional systems in the brain that are responsive to sensory signals; overlap between processes involved in mental imagery and perception which can lead to responding "as if" to real emotion-arousing events; and the capacity of images to make contact with memories for emotional episodes in the past [68-70].

Our finding provides insight into which category of words are highly associated with fear reactivity in males and females. In males imageability of mostly negative emotional words are highly associated with fear reactivity. Whereas in females, imageability of a wider range of word categories, that is, imageability of all negative words, all positive words, neutral and negative theoretical words, are associated with fear reactivity. This greater sensitivity to imageability across larger word categories and its association to fear reactivity in women serves as a plausible explanation to notion that women are more vulnerable to greater fear and anxiety in general, as opposed to men.

This greater sensitivity especially for imageability of all positive words may act as a protective factor against high fear reactivity in women. Only females, but not males, showed that having high imageability for positive words was associated with a lower fear reactivity. This provides insight into the potential coping strategies for anxious and fearful females. In fact, several studies have demonstrated that women tend to use emotionalfocused coping styles that are aimed at changing their emotional responses to a situation, as opposed to men who use more problem-focused or instrumental methods of handling anxious or stressful situations $[31,33,35]$. Hence, this could be a reason for why we do not observe the influence of the imageability of positive words in our male sample. Being able to reevoked perceptual representations of positive emotional words with better ease may induce the same effects as emotional-focused coping.

\section{Limitations and Future Directions}

Some parts of the tree model stratification are not clear-cut to explain. For example, in males, high imageability of negative propriosensitive word is associated with lower number of extreme fears and phobias, whereas low imageability of the same word category is associated with high number of extreme fears and phobias. This finding was in contrast to our hypothesis that postulated high imageability of negative word categories would be predictive of high fear reactivity. One plausible explanation is that negative propriosensitive words (e.g., 'spasm', 'convulsion', 'pale', 'burn', 'unease', 'nausea', etc.) was not directly associated to the fear tendency in different situations measured in this study. This part of the stratification amongst males in not clear-cut to interpret as we did not consider fear reactivity scores for different categories of fears; animal, social and interpersonal, health, noises, etc. Future studies could look to assess fear reactivity scores of such subcategories and its correlation to imageability, to obtain clear stratification and hence predictions.

Another limitation is that we used predefined ratings to categorize words into different categories. In females, greater imageability of neutral theoretical word 'convocation' is associated with fear tendency. This was also in contrast to our hypothesis that postulated high imageability of negative word categories, as opposed to a neutral word category seen here, would be predictive of high fear reactivity. Perhaps the word 'convocation' may not be perceived with a neutral valence but instead a negative valence amongst our sample of females. The thought of completing one's education may be stress-inducing. Hence, the lack of valence ratings of the words from our study sample poses another limitation. Having valence ratings of the words from the study sample instead of using predefined ratings, as well as arousal ratings, would help better understand this stratification. Additionally, having the valence and arousal ratings of all the words from the study sample itself would have helped understand any potential gender differences in the perception of these words. For future research, any differential affect based on gender towards the words should be considered. In this way, words included in each word category can be tailored specifically to males and females in order to ensure that males and females would perceive each word category (with same number of words) with a similar indented affect. For example, if 
the valence rating of the word 'convocation' is relatively neutral in males but negative in females, then 'convocation' should be considered a part of the neutral theoretical word category for males and negative theoretical word category for females. In this manner, male and female differences on specific wording can be controlled during the analyses.

It should be noted here that the individual words listed in the tree models predicting fear reactivity maybe specific to the sample in our study. Although the words may not be exactly the same for other samples, the category it belongs to may still be consistent across different study samples. In this study, only 171 participants with mean age of approximately 32 years took part in the study. Imageability ratings of different word categories should be administered to various age groups increasing the sample size in order to increase the generalizability of these findings across a larger population.

Although there were no outliers for fear tendency and extreme fear and phobia scores, we do not have any clinical diagnosis of anxiety or phobia for our study sample to confirm if any participant had clinical anxiety or phobia. Hence the results should be interpreted with caution when generalizing to general population.

A plausible explanation to gender differences found in this study could be due to differences in underlying neural mechanisms for fear imageability and fear reactivity in males and females. Imageability of different word categories could activate the fearnetworks differently in males and females [71,72]. This study sets stage for further research investigate the neural correlates of imageability and its association to fear reactivity to discover the any differential underlying neural mechanisms amongst males and females.

\section{Conclusions}

Despite the limitations, this study stands to be relevant from a clinical perspective since the imageability ratings of word categories denoting emotional, propriosensitive, and theoretical words can be associated with an individual's level of fear reactivity. Considering that people with fear reactivity fall on a spectrum, there are no specific markers that can easily predict where on the spectrum a person falls. Our study shows that a simple task such as rating mental imagery may be useful for performing a stratification of the population. This study provides an insight into how the possibility of controlling or enhancing relevant mental imagery, especially of that pertaining to emotional and theoretical word formation based on valence, may be efficient in reducing fearfulness or general anxiety.

Author Contributions: Conceived, designed, and performed the study: C.M., G.E. Analyzed the data: B.L.R., C.M., G.E. Interpreted results and wrote the paper: B.L.R., C.M., M.J.-Y.N., M.H.B., G.E. All authors have read and agreed to the published version of the manuscript.

Funding: This research was funded by the NAP-SUG program of the Nanyang Technological University (G.E.), the Intramural Research Program of the NIH/NICHD, USA (M.H.B.), and an International Research Fellowship at the Institute for Fiscal Studies (IFS), London, UK (M.H.B.), funded by the European Research Council (ERC) under the Horizon 2020 research and innovation programme (grant agreement No. 695300-HKADeC-ERC- 2015-AdG) (M.H.B.). The founder agencies had no role in the conceptualization, design, data collection, analysis, decision to publish, or preparation of the manuscript.

Institutional Review Board Statement: The study was approved by the Internal Ethical Committee of the University of Padova (protocol 1895 dated 01.04.2016), and was conducted according to the principles expressed in the Declaration of Helsinki. Informed consent was obtained from the participants.

Informed Consent Statement: Informed consent was obtained from all subjects involved in the study.

Data Availability Statement: The data presented in this study are available on request from the corresponding author.

Acknowledgments: All participants in this study are gratefully acknowledged. The authors also thank Sara Dellantonio and Remo Job for their assistance. 
Conflicts of Interest: The authors declare no conflict of interest. The funders had no role in the design of the study; in the collection, analyses, or interpretation of data; in the writing of the manuscript, or in the decision to publish the results.

\author{
Abbreviations \\ The following abbreviations are used in this manuscript: \\ TF degree of fearfulness towards different situations \\ EF Total number of extreme fears and phobias \\ FSS-II Fear Survey Schedule \\ CBA Cognitive Behavioural Assessment
}

\title{
References
}

1. Paivio, A. Mental Representations: A Dual Coding Approach; Oxford University Press: Oxford, UK, 1990.

2. Connell, L.; Lynott, D. Strength of perceptual experience predicts word processing performance better than concreteness or imageability. Cognition 2012, 125, 452-465. [CrossRef] [PubMed]

3. Richardson, A. Memory Imagery; Springer: Berlin/Heidelberg, Germany, 1969.

4. Paivio, A. Imagery and Language. In Imagery; Segal, S.J., Ed.; Academic Press: Cambridge, MA, USA, $1971 ;$ pp. 7-32. [CrossRef]

5. Dellantonio, S.; Mulatti, C.; Pastore, L.; Job, R. Measuring inconsistencies can lead you forward: Imageability and the x-ception theory. Front. Psychol. 2014, 5, 708. [CrossRef]

6. Barsalou, L.W. Perceptions of perceptual symbols. Behav. Brain Sci. 1999, 22, 637-660. [CrossRef]

7. Barsalou, L.W. Grounded Cognition. Annu. Rev. Psychol. 2008, 59, 617-645. [CrossRef] [PubMed]

8. Barsalou, L.W.; Niedenthal, P.M.; Barbey, A.K.; Ruppert, J.A. Social embodiment. Psychol. Learn. Motiv. 2003, 43, 43-92.

9. Barsalou, L.W.; Wiemer-Hastings, K. Situating Abstract Concepts, In Grounding Cognition: The Role of Perception and Action in Memory, Language, and Thought; Cambridge University Press: New York, NY, USA, 2005; pp. 129-163.

10. Prinz, J.J. Furnishing the Mind: Concepts and Their Perceptual Basis; MIT Press: Cambridge, MA, 2004.

11. Prinz, J.J. Gut Reactions: A Perceptual Theory of Emotion; Oxford University Press: Oxford, UK, 2004.

12. Kousta, S.T.; Vigliocco, G.; Vinson, D.P.; Andrews, M.; Del Campo, E. The representation of abstract words: Why emotion matters. J. Exp. Psychol. Gen. 2011, 140, 14-34. [CrossRef] [PubMed]

13. Vigliocco, G.; Meteyard, L.; Andrews, M.; Kousta, S. Toward a theory of semantic representation. Lang. Cogn. 2009, 1, 219-247. [CrossRef]

14. Paivio, A. Dual coding theory: Retrospect and current status. Can. J. Psychol. Can. Psychol. 1991, 45, 255. [CrossRef]

15. Paivio, A. Mind and Its Evolution: A Dual Coding Theoretical Approach; Lawrence Erlbaum Associates: Mahwah, NJ, USA, 2007.

16. Marks, D.F. Visual imagery differences in the recall of pictures. Br. J. Psychol. 1973, 64, 17-24. [CrossRef]

17. Katz, A.N. What does it mean to be a high imager. In Imagery, Memory and Cognition; Lawrence Erlbaum: Hillsdale, NJ, USA, 1983; pp. 39-63.

18. Riding, R. Cognitive styles analysis-CSA administration. Birm. Learn. Train. Technol. 1991.

19. Peterson, E.R.; Deary, I.J.; Austin, E.J. A New Measure of Verbal-Imagery Cognitive Style: VICS. Pers. Individ. Differ. 2005, 38, 1269-1281. [CrossRef]

20. Aron, E.N.; Aron, A.; Jagiellowicz, J. Sensory processing sensitivity: A review in the light of the evolution of biological responsivity. Pers. Soc. Psychol. Rev. 2012, 16, 262-282. [CrossRef] [PubMed]

21. Grimen, H.L.; Diseth, A. Sensory processing sensitivity: Factors of the highly sensitive person scale and their relationships to personality and subjective health complaints. Compr. Psychol. 2016, 5, 2165222816660077. [CrossRef]

22. Gullone, E. The development of normal fear. Clin. Psychol. Rev. 2000, 20, 429-451. [CrossRef]

23. McCathie, H.; Spence, S.H. What is the revised Fear Survey Schedule for Children measuring? Behav. Res. Ther. 1991, $29,495-502$. [CrossRef]

24. Wolpe, J.; Lang, P.J. A fear survey schedule for use in behaviour therapy. Behav. Res. Ther. 1964, 2, 27-30. [CrossRef]

25. Ferguson, E.; Moghaddam, N.G.; Bibby, P.A. Memory bias in health anxiety is related to the emotional valence of health-related words. J. Psychosom. Res. 2007, 62, 263-274. [CrossRef]

26. Ahadi, B.; Basharpoor, S. Relationship Between Sensory Processing Sensitivity, Personality Dimensions and Mental Health. J. Appl. Sci. 2010, 10, 570-574. [CrossRef]

27. Aron, E.N.; Aron, A. Sensory-processing sensitivity and its relation to introversion and emotionality. J. Pers. Soc. Psychol. 1997, 73, 345-368. [CrossRef]

28. Hofmann, S.G.; Bitran, S. Sensory-processing sensitivity in social anxiety disorder: relationship to harm avoidance and diagnostic subtypes. J. Anxiety Disord. 2007, 21, 944-954. [CrossRef]

29. Liss, M.; Timmel, L.; Baxley, K.; Killingsworth, P. Sensory processing sensitivity and its relation to parental bonding, anxiety, and depression. Personal. Individ. Differ. 2005, 39, 1429-1439. [CrossRef] 
30. Liss, M.; Mailloux, J.; Erchull, M.J. The relationships between sensory processing sensitivity, alexithymia, autism, depression, and anxiety. Personal. Individ. Differ. 2008, 45, 255-259. [CrossRef]

31. Endler, N.S.; Parker, J.D.A. State and trait anxiety, depression and coping styles. Aust. J. Psychol. 1990, 42, 207-220. [CrossRef]

32. Fredrikson, M.; Annas, P.; Fischer, H.; Wik, G. Gender and age differences in the prevalence of specific fears and phobias. Behav. Res. Ther. 1996, 34, 33-39. [CrossRef]

33. Matud, M.P.; Pilar Matud, M. Gender differences in stress and coping styles. Personal. Individ. Differ. 2004, 37, 1401-1415. [CrossRef]

34. McLean, C.P.; Anderson, E.R. Brave men and timid women? A review of the gender differences in fear and anxiety. Clin. Psychol. Rev. 2009, 29, 496-505. [CrossRef]

35. Kelly, M.M.; Tyrka, A.R.; Anderson, G.M.; Price, L.H.; Carpenter, L.L. Sex differences in emotional and physiological responses to the Trier Social Stress Test. J. Behav. Ther. Exp. Psychiatry 2008, 39, 87-98. [CrossRef]

36. Vambheim, S.M.; Øien, R.A. Sex differences in fear of pain: Item-level analysis of the Fear of Pain Questionnaire III. J. Pain Res. 2017, 10, 825. [CrossRef] [PubMed]

37. Wassenburg, S.I.; de Koning, B.B.; de Vries, M.H.; Boonstra, A.M.; van der Schoot, M. Gender differences in mental simulation during sentence and word processing. J. Res. Read. 2017, 40, 274-296. [CrossRef]

38. Campos, A. Gender differences in imagery. Personal. Individ. Differ. 2014, 59, 107-111. [CrossRef]

39. Grandy, T.H.; Lindenberger, U.; Schmiedek, F. Vampires and nurses are rated differently by younger and older adults-Agecomparative norms of imageability and emotionality for about 2500 German nouns. Behav. Res. Methods 2020, 52, 980-989. [CrossRef]

40. Esposito, G.; Dellantonio, S.; Mulatti, C.; Job, R. Axiom, Anguish, and Amazement: How Autistic Traits Modulate Emotional Mental Imagery. Front. Psychol. 2016, 7, 757. [CrossRef] [PubMed]

41. Vigliocco, G.; Vinson, D.P.; Druks, J.; Barber, H.; Cappa, S.F. Nouns and verbs in the brain: A review of behavioural, electrophysiological, neuropsychological and imaging studies. Neurosci. Biobehav. Rev. 2011, 35, 407-426. [CrossRef]

42. Tomkins, S.S. Affect Imagery Consciousness: Volume I: The Positive Affects; Springer Publishing Company: New York, NY, USA 1962.

43. Tomkins, S. Affect Imagery Consciousness: Volume II: The Negative Affects; Publishing Company: New York, NY, USA, 1963; Volume 2.

44. Ekman, P. Expression and the Nature of Emotion in Approachs to Emotion. Approaches Emot. 1984, 3, 344.

45. Ekman, P. Moods, emotions, and traits. In The Nature of Emotion: Fundamental Questions; Oxford University Press: Oxford, UK, 1994; pp. 56-58.

46. Ekman, P. Basic Emotions. Handb. Cogn. Emot. 1999, 98, 16.

47. Plutchik, R. Psychoevolutionary Theory of Basic Emotions. Soc. Sci. Inf. 1980, 21, 529-553.

48. Reisenzein, R. Emotional Experience in the Computational Belief-Desire Theory of Emotion. Emot. Rev. 2009, 1, 214-222. [CrossRef]

49. Kassam, K.S.; Markey, A.R.; Cherkassky, V.L.; Loewenstein, G.; Just, M.A. Identifying Emotions on the Basis of Neural Activation. PLoS ONE 2013, 8, e66032. [CrossRef] [PubMed]

50. Damasio, A.R. The Feeling of What Happens: Body and Emotion in the Making of Consciousness; Harcourt Brace: New York, NY, USA, 1999.

51. Berthoz, A. The Brain's Sense of Movement;Harvard University Press: Cambridge, MA, USA, 2000.

52. Craig, A.D. Interoception: The sense of the physiological condition of the body. Curr. Opin. Neurobiol. 2003, 13, 500-505. [CrossRef]

53. Craig, A.D.; Craig, A.D. How do you feel—now? The anterior insula and human awareness. Nat. Rev. Neurosci. 2009, 10, 59-70. [CrossRef] [PubMed]

54. Craig, A.D.B. The sentient self. Brain Struct. Funct. 2010, 214, 563-577. [CrossRef]

55. Warriner, A.B.; Kuperman, V.; Brysbaert, M. Norms of valence, arousal, and dominance for 13,915 English lemmas. Behav. Res. Methods 2013, 45, 1191-1207. [CrossRef]

56. Sanavio, E.; Bertolotti, G.; Michielin, P.; Vidotto, G.; Zotti, A. CBA-2.0 Scale primarie; Organizzazioni Speciali: Firenze, Italy, 1986.

57. Sanavio, E. CBA-2.0: Cognitive Behavioural Assessment 2.0: Scale Primarie: Manuale; Organizzazioni Speciali: Firenze, Italy, 1997.

58. Paivio, A.; Yuille, J.C.; Madigan, S.A. Concreteness, imagery, and meaningfulness values for 925 nouns. J. Exp. Psychol. 1968, 76, 1-25. [CrossRef]

59. Pastore, L.; Dellantonio, S.; Mulatti, C.; Job, R. On the Nature and Composition of Abstract (Theoretical) Concepts: The X-Ception Theory and Methods for Its Assessment. In Philosophy and Cognitive Science II; Springer: Cham, Switzerland, 2015.

60. Fox, A.S.; Lapate, R.C.; Shackman, A.J.; Davidson, R.J. The Nature of Emotion: Fundamental Questions; Oxford University Press: Oxford, UK, 2018.

61. Costello, T.J.; Swartz, M.D.; Sabripour, M.; Gu, X.; Sharma, R.; Etzel, C.J. Use of tree-based models to identify subgroups and increase power to detect linkage to cardiovascular disease traits. BMC Genet. 2003, 4 (Suppl. 1), S66. [CrossRef]

62. Esposito, G.; Manian, N.; Truzzi, A.; Bornstein, M.H. Response to infant cry in clinically depressed and non-depressed mothers. PLoS ONE 2017, 12, e0169066. [CrossRef] [PubMed]

63. Fox, J. Applied Regression Analysis and Generalized Linear Models; SAGE Publications: Thousand Oaks, CA, USA, 2015. 
64. Mobini, S.; Grant, A. Clinical implications of attentional bias in anxiety disorders: An integrative literature review. Psychother. Theory, Res. Pract. Train. 2007, 44, 450. [CrossRef] [PubMed]

65. Becker, E.S.; Rinck, M.; Margraf, J.; Roth, W.T. The emotional Stroop effect in anxiety disorders. J. Anxiety Disord. 2001, 15, 147-159. [CrossRef]

66. Johnsen, B.H.; Thayer, J.F.; Laberg, J.C.; Wormnes, B.; Raadal, M.; Skaret, E.; Kvale, G.; Berg, E. Attentional and physiological characteristics of patients with dental anxiety. J. Anxiety Disord. 2003, 17, 75-87. [CrossRef]

67. Owens, K.M.B.; Asmundson, G.J.G.; Hadjistavropoulos, T.; Owens, T.J. Attentional Bias Toward Illness Threat in Individuals with Elevated Health Anxiety. Cogn. Ther. Res. 2004, 28, 57-66. [CrossRef]

68. Holmes, E.A.; Mathews, A. Mental imagery in emotion and emotional disorders. Clin. Psychol. Rev. 2010, 30, 349-362. [CrossRef]

69. Holmes, E.A.; Lang, T.J.; Shah, D.M. Developing interpretation bias modification as a "cognitive vaccine" for depressed mood: imagining positive events makes you feel better than thinking about them verbally. J. Abnorm. Psychol. 2009, 118, 76-88. [CrossRef] [PubMed]

70. Ohman, A.; Mineka, S. Fears, phobias, and preparedness: Toward an evolved module of fear and fear learning. Psychol. Rev. 2001, 108, 483-522. [CrossRef] [PubMed]

71. Lai, C.H. Fear network model in panic disorder: The past and the future. Psychiatry Investig. 2019, 16, 16. [CrossRef] [PubMed]

72. Shin, L.M.; Liberzon, I. The neurocircuitry of fear, stress, and anxiety disorders. Neuropsychopharmacology 2010, 35, 169-191. [CrossRef] [PubMed] 